\title{
On the Applicability of Exact Optimization in Search Based Software Engineering
}

\author{
Fabrício Freitas, Thiago Silva, Rafael Carmo, and Jerffeson Souza \\ Optimization in Software Engineering Group (GOES.UECE) \\ Natural and Intelligent Computing Lab (LACONI) \\ State University of Ceará (UECE) - Avenue Paranjana, 1700, Ceará, Brazil \\ \{fabriciogf.uece, thi.nepo, carmorafael\}@gmail.com, \\ jeffalarces.uece.br
}

\begin{abstract}
The Search Based Software Engineering (SBSE) field has emerged as an exciting and promising area by proposing the formulation of software engineering problems as optimization problems. Hitherto, metaheuristics have been widely employed for solving these problems, whilst little work has been done regarding the use of exact techniques in the area. This paper aims to fulfil this lack by presenting a comprehensive study on the theory and practice of the application of exact optimization in SBSE. A conceptual comparison of both optimization approaches in the software engineering context is presented. Problems' aspects are analysed regarding suitability for use of exact techniques. As illustration, comparison experiments with exact technique and metaheuristics are conducted over a well-known SBSE problem. The results reveal the overall behaviour of exact techniques, regarding efficacy and efficiency, in the SBSE context considered, indicating its potential use.
\end{abstract}

Keywords: exact optimisation, theoretical work, empirical study, SBSE applications, optimisation techniques comparison. 